\title{
Notifire: A Microcontroller (Arduino) Operated Device for Early Fire Detection and Risk Reduction
}

\author{
${ }^{1}$ Marco Antonio A. Galo, ${ }^{2}$ Kyle A. De La Torre, ${ }^{3}$ Rizelin John Ofelia \\ ${ }^{123} \mathrm{HS}$ Students, \\ Philippine Science High School-Central Visayas Campus \\ Talaytay, Argao, Cebu
}

\begin{abstract}
Fire can be either an extremely useful tool in our daily lives or a dangerous existence in itself that results to disasters that often cost more than money. Fire incidents are often the ones that generate the most damage in a short amount of time due to it being rapid and leave no other salvageable parts. To combat these, early fire detection and prevention devices have been invented and made. In this research, an early fire detection device has been made along with a message sender response. The device has been made to detect temperature, gas and flame, which are possible signs of a fire. The device consists of the circuit containing an Arduino module, GSM module for message sending, sensors, buzzer and LEDs, and an acrylic casing to house the circuit. The device detected an average maximum distance range of $8.33 \mathrm{~cm}$ for gas, $177.33 \mathrm{~cm}$ for flame and 2.43 $\mathrm{cm}$ for temperature. The device also has successfully sent text message to designated cellphone number indicating the situations the device has detected such as high temperature and gas leak. A survey was performed for feedbacks about the usability, aesthetics and functionality of the device and results showed a possible marketability potential with a Net Promoter Score of $33 \%$. Overall, the device can serve as a functional early fire detection device based on tests and surveys.
\end{abstract}

Keywords—NotiFire; Fire; Fire Detection; Arduino; GSM;

\section{INTRODUCTION}

Fire can either be an extremely useful tool in our daily lives or a dangerous existence in itself that results to disasters that often cost more than money [1] Handled carelessly, fire can be as destructive as it can be useful. Fires are considered as a major security concern around the globe. Fire incidents are often the ones that generate the most damage in a short amount of time, leaving no salvageable parts [2] Fire destroys properties and resources, and can even extinguish life. The 2015 Annual Accomplishment Report by the Bureau of Fire Protection of the Philippines, estimates that almost 3.62 billion pesos worth of properties have been damaged by fire and a total number of 1049 people were recorded with casualties or injuries related to it. These data also show an increase of $9.70 \%$ for recorded property damage and a significant increase for reported casualties and injuries compared to 2014.

Fire detectors are technologies commonly used to detect and prevent fire outbreaks. A fire detector detects and responds to the signs of fire such as smoke, heat and radiation, thereby preventing or minimizing the damage done by fire. Humans, in fact, are fire detectors with their five senses. Their actions warn nearby people about their perceived information about the fire and their actions are made through their processed thoughts. This is called manual fire detection and is one of the oldest methods of fire detection [3].

Automatic fire detection devices were made in order to minimize human error as much as possible and to respond accordingly even without the presence of humans. The oldest type of these devices was thermal detectors during the mid 1800's. In general, automatic fire detection devices/systems operate by having a set of algorithm that responds to the fire. For example, thermal detectors operate when a pre-determined temperature is sensed and recorded. After detecting the specified value, sprinklers will activate in order to extinguish the fire or at least minimize its damage. Newer ones such as smoke detectors identify a fire through the signs of smoke. They operate on the systems of photoelectric principle, air aspiration or ionization and alarm the surrounding area that there is a fire [3]. Along these detectors are various response mechanisms that adapt through situations and have helped prevent fires and solve fire-related problems

This study will incorporate the use and study the feasibility of microcontrollers in fire detection systems. The main goal of the study is to fabricate a reliable (as based on its accuracy and precision) yet low-maintenance fire detection system using sensors readily available in the market in order to address the challenge of maintaining home security.

\section{OBJECTIVES}

- Fabricate an early fire detection device controlled by an Arduino microcontroller using flame, gas, and temperature sensors compatible with it;

- Evaluate the performance of the device by measuring its maximum range of detection in a simulated environment for flame, gas, and temperature sensors; and

- Evaluate the device through external evaluators (respondents) through a standard formulated questionnaire comprising queries relating to customer feedback. 


\section{FABRICATION OF THE DEVICE}

\section{A. Components of the Device}

- Arduino ${ }^{\circledR}$ Uno- an open source electronics microcontroller platform used for prototyping and various electronics purposes[4]

- Flame sensor - a variation of the infrared (IR) sensor that is set to detect only a range of $760 \mathrm{~nm}$ to 1100 $\mathrm{nm}[5]$

- GSM- stands for Global System for Mobile communication, a type of communication network that is widely used

- LM35 temp sensor -a precision integrated circuit type of temperature sensor for Arduino

- MQ2 Gas Sensor- a flammable gas detector for Arduino

- LED- stands for light emitting diode, a type of diode that emits light upon flow of current

\section{B. Design of the Circuit}

The circuit of the device was designed to ensure efficiency for the device in terms of space and placement of components and the circuit itself. The Arduino device, along with the GSM module, LED and buzzer, gas, temperature, and flame detector, was attached to the circuit according to Fig 1.The sensors detected changes in parameters that they are assigned to measure. When one of these sensors measures an abnormality that points to a fire (e.g. high temperature and gas leak), the buzzer, and the corresponding LED will activate, and the GSM module will send a distress message. The GSM and Arduino will be powered by separate $5 \mathrm{~V}$ sources.

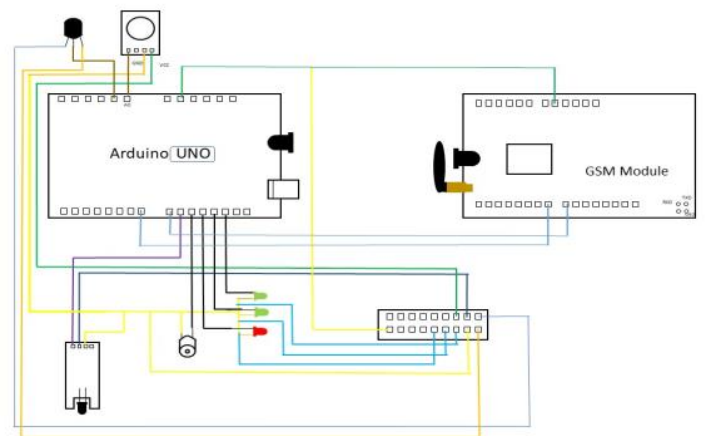

Fig 1: Circuit Design for the Device

\section{Encasing the Circuitry}

The circuit was placed inside a compartment made from acrylic sheets. The compartment had holes and spaces allotted for each sensor and the outlet for the power source. The device was then sealed and furnished with having one side of the compartment be made removable for future modifications of the circuit.

\section{Testing of Device}

The device was tested using a flame source both for heat and flame parameters and a small butane gas in order to determine if the sensors were working and were integrated properly with the GSM module. The maximum range of detection for each of the sensor was taken by moving the source of fire away from the sensor in increments of $1 \mathrm{~cm}$ until the device could not pick up a reading for 5 seconds. Afterwards, the source was moved closer between the $1 \mathrm{~cm}$ ranges where the fire has been last detected to determine the precise value. The algorithm of the device is as shown in Fig 2.

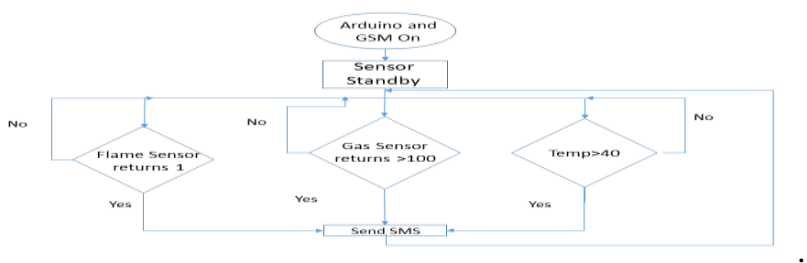

Fig 2: Algorithm of NotiFire

\section{E. User Satisfaction Survey}

After the device was tested in its prototype stage and finalized, a user satisfaction survey was done covering the aesthetics, functionality and the willingness to use and recommend the device.A rating scale from 0 to 10 was used where 0 was the lowest score (not at all likely), 5 being neutral, and 10 being the highest score (extremely likely, patterned after Robert Rubinoff's "Quantifying User Experience" and Net Promoter Score formats. It was done by random sampling to get the 30 respondents from Philippine Science High School - Central Visayas Campus. The Net Promoter Score (NPS) formula utilized for the analysis is given by the following [6].

$N P S=[\%$ Promoters with 9 s to10s] $-[\%$ Detractors with 0 s to $5 s]$

\section{RESULTS}

A. Fabrication of the Final Device

The circuit component of the device was assembled and placed in an acrylic casing to fabricate the device. The acrylic casing have been bore with holes for the circuit parts to go through such as the sensors, LEDs, the GSM antenna and the power cord. The device was then furnished as shown in Fig 3.

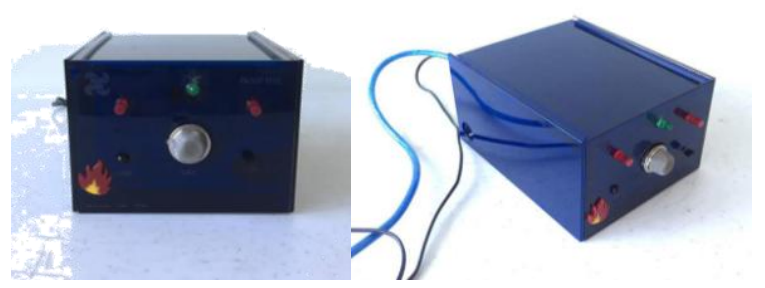

Fig 3: The NotiFire Device 


\section{B. Fire Detection Sensors and Response}

The sensors of the device were tested individually to verify if they were properly working or not and the maximum distance ranges it can detect the source at a given time for a particular sensor was determined. The testing shows that all of the sensors are working as each sensor's corresponding LED lit up during stimulus. A buzzer connected to the circuit also emits a sound which indicates that the source has been detected by the device.

Each sensor's maximum distance range was tested and recorded in a total of three trials per sensor. As shown in Table 1 , the flame sensor has an average maximum distance range of $1.17 \mathrm{~m}$ while the gas sensor has an average maximum distance of less than $10 \mathrm{~cm}$. The maximum detection range for the temperature sensor was rather small compared to the other sensors. This may be due to the fact that the said sensor measures the ambient room temperature.

TABLE 1. Maximum distance range of the sensors

\begin{tabular}{|c|c|c|c|c|}
\hline Sensor & Trial 1 & Trial 2 & Trial 3 & Average \\
\hline Gas & $\mathbf{9 . 0} \mathbf{~ c m}$ & $\mathbf{8 . 0} \mathbf{~ c m}$ & $\mathbf{8 . 0} \mathbf{~ c m}$ & $\mathbf{8 . 3 \pm 0 . 6} \mathbf{~ c m}$ \\
\hline Flame & $\mathbf{1 2 0} \mathbf{~ c m}$ & $\mathbf{1 1 5} \mathbf{~ c m}$ & $\mathbf{1 1 7} \mathbf{~ c m}$ & $\mathbf{1 1 7 \pm 3 c m}$ \\
\hline Temperature & $\mathbf{2 . 4} \mathbf{~ c m}$ & $\mathbf{2 . 4} \mathbf{~ c m}$ & $\mathbf{2 . 5} \mathbf{~ c m}$ & $\mathbf{2 . 4 \pm 0 . 1} \mathbf{~ c m}$ \\
\hline
\end{tabular}

\section{Device Message Response}

The device has been successfully programmed to send a message to a designated cellphone number using the GSM module when it detects the source of fire. Each sensor sends a different message when different indicators of fire are detected. Each message depends on the data received and sent by the device as shown in Fig 4.

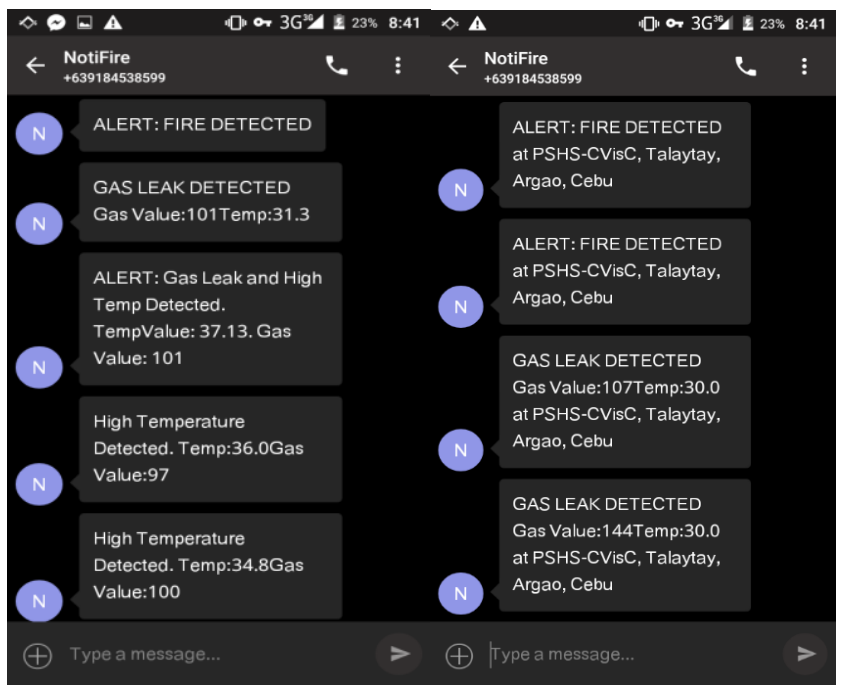

Fig 4: Sample message response from NotiFire

\section{User Satisfaction Survey}

A total of 30 respondents from Philippine Science High School - Central Visayas Campus were surveyed. Each respondent gave the device ratings in terms of usability, aesthetics and functionality and were recorded. The average scores of the survey were tabulated and a net promoter score calculation was performed.
TABLE 2. Average Scores of the Device

\begin{tabular}{lc}
\hline Category & $\begin{array}{c}\text { Average } \\
\text { Score }\end{array}$ \\
\hline Usability & 8.3 \\
Aesthetics & 7.6 \\
Functionality & 8.1 \\
\hline
\end{tabular}

As shown in Table 2, the score in each category ranges between 6-8. The device's usability has the highest average score of 8.3 , functionality having an average score of 8.1 while its aesthetics has the lowest of 7.6. The average scores were then sorted and classified according to their ranges with detractors as having average score range of $0-6$, passives with 6-8, and promoters with 9-10. Frequencies of each respondent classification were noted and are shown in the figure below to determine the Net Promoter Score.

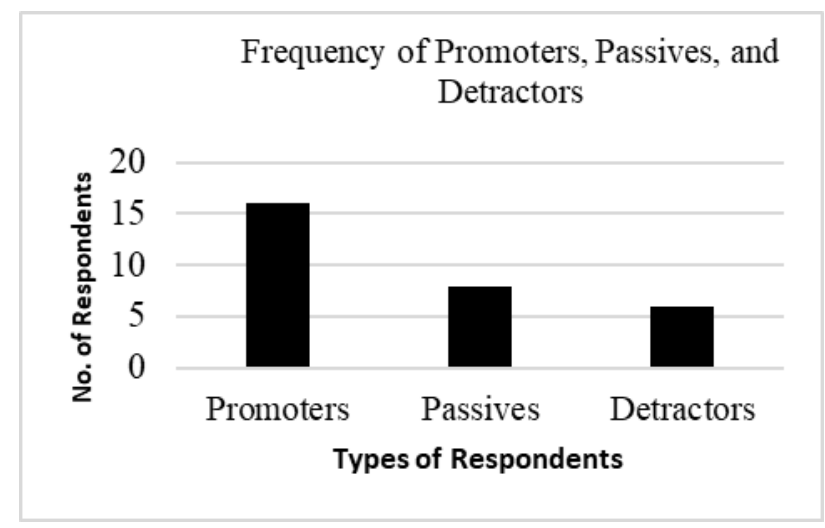

Fig 5. Frequency of Promoters, Passives, and Detractors

Survey results shows that among the 30 respondents there were 16 promoters, 8 passives and 6 detractors. The calculated net promoter score of the device is $33 \%$ with $20 \%$ detractors, $27 \%$ passives and $53 \%$ promoters. This shows that greater than half of the population promotes the device and a fifth population demotes it while the rest of the respondents are having neutral thoughts about it resulting in a miniscule possible potential for marketability.

\section{CONCLUSIONS}

An early fire detection device was successfully developed. The fabricated device, controlled by an Arduino microcontroller using flame, gas and temperature sensors, had its performance successfully evaluated in terms of its maximum range of detection in a simulated environment. The maximum range of detection for the flame, temperature, and gas sensors are $117 . \mathrm{cm}, 2.43 \mathrm{~cm}$, and $8.33 \mathrm{~cm}$ respectively. From the user satisfaction survey, the device was evaluated with an average score of 8.3 for usability, 8.1 for functionality and 7.6 for its aesthetics showing that the device is evaluated more on its usability and functionality. The device got a net promoter score of $33 \%$ that suggests a possible potential for marketability if the aesthetics is greatly improved. 


\section{ACKNOWLEDGMENT}

The researchers would like to thank as a group Mr. Jayfe Anthony A. Abrea for providing intellectual support for the research, Mr. Benito A. Baje for his unending support both intellectually and morally, Mr. Paul Isaac Dizon for being the panel of this paper, Mr. Eleazar B. Guia for coordinating the panel and expediting the completion of the paper, and Mrs. Arlene V. Cahoy-Agosto for her careful review of the paper as the research teacher. The researchers would also like to thank their friends and family for their support.

\section{REFERENCES}

[1] Dehesa, T. (2015). Common Causes of Fire in Pinoy Homes. Retrieved November 28, 2017, from Real Living: realliving.com.ph

[2] Bahrudin, M. S., Buniyamin, N., \& Kassim, R. A. (2013). Development of Fire Alarm System using Raspberry $\mathrm{Pi}$ and Arduino Uno. International Conference on \ Electrical, Electronics and System Engineering , 43-48.I.S. Jacobs and C.P. Bean, "Fine particles, thin films and exchange anisotropy," in Magnetism, vol. III, G.T. Rado and H. Suhl, Eds. New York: Academic, 1963, pp. 271-350.

[3] Artim, N. (2015). An Introduction to Fire Detection, Alarm, and Automatic Fire \Sprinklers.

[4] Ishikawa, M., Maruta, I. (2009). Rapid Prototyping for Control Education Using Arduino and Open-Source Technologies

[5] Future Electronics Egypt Ltd. . (2017, November 29). Flame Sensor Module. Egypt

[6] Rubinoff, Robert (2004). How to quantify the user experience

[7] A. Araujo, D. Portugal, M. Couceiro, R. Rocha (2013), Intergrating Arduino-based Educational Mobile Robots in ROS. Gulf Medical Journal. 3(S1):S101-S113R. Nicole, "Title of paper with only first word capitalized," J. Name Stand. Abbrev., in press.

[8] National Semiconductor. (2000). LM35 Precision Centigrade Temperature Sensors. 